\title{
Clinical utility of 18-fluorodeoxyglucose positron emission tomography/computed tomography in malignant pleural mesothelioma
}

\author{
TAKAYUKI TERADA ${ }^{1,2^{*}}$, CHIHARU TABATA ${ }^{1,2^{*}}$, RIE TABATA $^{3}$, HISAYA OKUWA $^{1}$, \\ SHINGO KANEMURA ${ }^{1}$, EISUKE SHIBATA ${ }^{1}$ and TAKASHI NAKANO ${ }^{1,2}$ \\ ${ }^{1}$ Division of Respiratory Medicine, Department of Internal Medicine, Hyogo College of Medicine, \\ ${ }^{2}$ Department of Thoracic Oncology, Hyogo College of Medicine, ${ }^{3}$ Department of Internal Medicine, \\ Hyogo Prefectural Tsukaguchi Hospital, Hyogo, Japan
}

Received March 6, 2012; Accepted May 5, 2012

DOI: $10.3892 /$ etm.2012.572

\begin{abstract}
Malignant pleural mesothelioma (MPM) is an aggressive malignant tumor of mesothelial origin associated with asbestos exposure. MPM has a limited response to conventional chemotherapy and radiotherapy, thus early diagnosis of MPM is extremely critical. CT scans have limited accuracy in the differentiation between benign and malignant pleural disease. Several studies have reported that 18-fluorodeoxyglucose (FDG) positron emission tomography (PET) plays an important role in the assessment of thoracic malignancy such as lung cancer. Here, we investigated the clinical utility of PET in patients with MPM. The maximum SUV (SUVmax) of 18F-FDG was measured in 47 MPM patients and 29 non-MPM patients including those with pleural thickening. We demonstrated that patients with MPM had significantly higher SUVmax levels than a population with non-malignant pleural disease. The Kaplan-Meier method revealed significant differences in overall survival between groups with SUVmax levels lower and higher than the assumed cut-off. Our data suggest that SUVmax levels are useful as an aid for diagnosis and prognosis of MPM.
\end{abstract}

Correspondence to: Dr Chiharu Tabata, Division of Respiratory Medicine, Department of Internal Medicine, Hyogo College of Medicine, 1-1 Mukogawa-cho, Nishinomiya, Hyogo 663-8501, Japan E-mail: ctabata@hyo-med.ac.jp

*Contributed equally

Abbreviations: AUC, area under the ROC curve; CI, confidence interval; CT, computed tomography; FDG, fluorodeoxyglucose; MRI, magnetic resonance imaging; MPM, malignant pleural mesothelioma; NPV, negative predictive value; PET, positron emission tomography; PPV, positive predictive value; ROC, receiver operating characteristic; ROI, region of interest; SUVmax, maximum SUV; VATS, videoassisted thoracic surgery

Key words: pleural mesothelioma, SUVmax, diagnosis, prognosis

\section{Introduction}

Malignant pleural mesothelioma (MPM) is an aggressive malignant tumor of mesothelial origin associated with asbestos exposure (1-3). Although asbestos usage has recently been banned in Western countries and Japan, the incidence of MPM is expected to markedly increase over the next few decades since there is a long latency period (20-40 years) between asbestos exposure and tumor development (4). MPM shows limited response to conventional chemotherapy and radiotherapy. Although multi-targeted anti-folate pemetrexed has been approved as a first-line agent in combination with cisplatin for MPM treatment, overall survival remains very poor (5) with median survival durations of 8-18 months (6). In several centers, potentially curative surgery combined with some form of adjuvant therapy has been performed. Such early therapeutic intervention appears to be more beneficial than late intervention. Diagnosing MPM is critical (1) since the general conditions of these patients such as a poor performance status may hinder adequate therapy. However, diagnosis may be extremely difficult in histological studies.

Computed tomography (CT) plays a role in identifying the location and dissemination of malignant pleural tumors (7), however, it is not always able to differentiate between malignant and benign pleural lesions (8). Pleural biopsies such as video-assisted thoracic surgery (VATS) are required to enable definite diagnosis of MPM. However, these are invasive procedures; therefore, new non-invasive techniques for assessment of MPM are required to judge whether those procedures should be practiced for diagnosis of MPM.

Currently, 18-fluorodeoxyglucose (FDG) positron emission tomography (PET) is an important imaging tool for the diagnostic assessment of patients with cancer (9). PET is useful for detecting malignant lung nodules $(10,11)$. There have been several reports concerning the uptake of FDG in MPM and clinical assessment such as diagnostic and prognostic information of MPM using PET (12-15). However, its clinical utility in MPM has not been fully investigated. In this study, we evaluated the diagnostic and prognostic role of PET in Japanese MPM patients. 


\section{Materials and methods}

Patients. This study was performed using 76 patients who presented at the Department of Respiratory Medicine of Hyogo College of Medicine Hospital from September 2009 to April 2011. Forty-seven individuals had malignant plural mesothelioma (MPM); 43 individuals were diagnosed using histopathological samples and 4 were diagnosed using cytological samples by pathologists skilled in the diagnosis of MPM. Thirty-two patients had a documented history of asbestos exposure. All 47 patients were classified using the staging system of the International Mesothelioma Interest Group (IMIG) (16). Patients with MPM were treated according to our therapeutic guidelines; combination chemotherapy including multi-target anti-folate pemetrexed or pemetrexed alone was administered to patients with PS 0-1, and the best supportive care was chosen for the remaining patients. Surgical and radiation treatment was performed on 3 patients in the present study. Twenty-nine individuals, including 3 cases with benign asbestos pleurisy, had non-malignant pleural effusion. We verified asbestos exposure by interview. Thirteen patients had a documented history of asbestos exposure. Informed consent was obtained from all patients. This study was approved by our ethics committee in accordance with the 1975 Declaration of Helsinki.

FDG-PET imaging. 18F-FDG PET/CT imaging was performed at the PET Center of Hyogo College of Medicine Hospital. All patients fasted for at least $6 \mathrm{~h}$ before PET/CT examination and their blood glucose concentrations were measured. Patients were scanned on a PET/CT scanner (GEMINI GXL 16; Philips Medical System, Eindhoven, The Netherlands) 60 min after injection of 18F-FDG depending on the patient's weight. CT was performed from head to foot at a $5.0-\mathrm{mm}$ slice thickness. For semi-quantitative assessment, regions of interest (ROIs) were examined. The maximum SUV (SUVmax) of 18F-FDG was measured from ROI according to the standard formula. PET and CT datasets were reported by two independent readers.

Statistical analysis. The nonparametric Mann-Whitney U-test was used to compare two groups. In all tests, a p-value $<0.05$ was considered to indicate statistical significance. In order to estimate the significance of SUVmax, receiver operating characteristic (ROC) curves, area under the ROC curves (AUC), and their $95 \%$ confidence intervals $(95 \% \mathrm{CI})$ were calculated using standard techniques. To obtain appropriate SUVmax level cut-off values, we calculated the total sensitivity and specificity for each cut-off value and then chose the cut-off values that maximized the sum of sensitivity plus 1-specificity. Estimates of the probability of survival were calculated using the KaplanMeier method and compared using the log-rank test. In order to evaluate SUVmax prognostic significance on the survival of patients with MPM, Cox's proportional hazards regression analysis (backward) was carried out as multivariate analysis.

\section{Results}

SUVmax levels in patients with MPM and patients with non-MPM. We recruited a total of 76 subjects. Of them, 47 had confirmed MPM and 29 had non-malignant pleural effusion.
Table I. Characteristics of the study groups.

\begin{tabular}{lr} 
A, MPM patients $(\mathrm{n}=47)$ & \\
\hline Patient characteristics & $\mathrm{n}(\%)$ \\
\hline Age (mean age \pm SD), in years & $65.2 \pm 9.6$ \\
Gender & \\
Male & $38(80.9)$ \\
Female & $9(19.1)$ \\
Histology & \\
Epithelioid & $31(66.0)$ \\
Sarcomatoid & $6(12.8)$ \\
Biphasic & $4(8.5)$ \\
Desmoplastic & $1(2.1)$ \\
Unknown & $5(10.6)$ \\
Stage & \\
I & $9(19.2)$ \\
II & $10(21.3)$ \\
III & $9(19.2)$ \\
IV & $19(40.3)$ \\
\end{tabular}

B, Non-malignant patients $(\mathrm{n}=29)$

\begin{tabular}{lr}
\hline Patient characteristics & $\mathrm{n}(\%)$ \\
\hline Age (mean age \pm SD), in years & $70.1 \pm 11.1$ \\
Gender & \\
Male & $22(75.9)$ \\
Female & $7(24.1)$ \\
CT findings & \\
Benign asbestos pleurisy & $3(10.3)$ \\
Plaque & $5(17.2)$ \\
Tuberculosis (TB) pleurisy & $1(3.5)$ \\
Infectious (non-TB) pleurisy & $1(3.5)$ \\
Chronic pleurisy & $6(20.7)$ \\
Pleural thickening & $12(41.3)$ \\
Pleural effusion & $1(3.5)$ \\
\hline
\end{tabular}

MPM, malignant pleural mesothelioma.

Their characteristics are shown in Table I. Of the 47 patients with MPM, 31 were of epithelioid histology, 6 sarcomatoid, 4 biphasic, 1 desmoplastic and 5 unknown.

The ROC curves for SUVmax levels demonstrated that patients with MPM had an AUC of 0.803 which differed from those with non-MPM (95\% CI, 0.722-0.885). At the optimal cut-off value of 3.5, the diagnostic sensitivity was $59.6 \%$, and the specificity was $93.1 \%$ (Fig. 1A). The positive predictive value (PPV) was $93.3 \%$, and the negative predictive value (NPV) was 58.7\%. The SUVmax level of patients with MPM was significantly higher $(5.3 \pm 4.4)$ than that of the non-MPM patients including those with benign asbestos pleurisy $(1.2 \pm 1.8)$ $(p<0.01)$ (Fig. 1B). Differences in SUVmax levels between the various MPM histological groups were significant (non- 
A

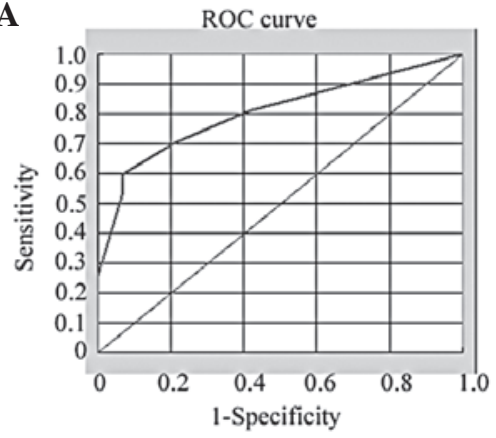

C

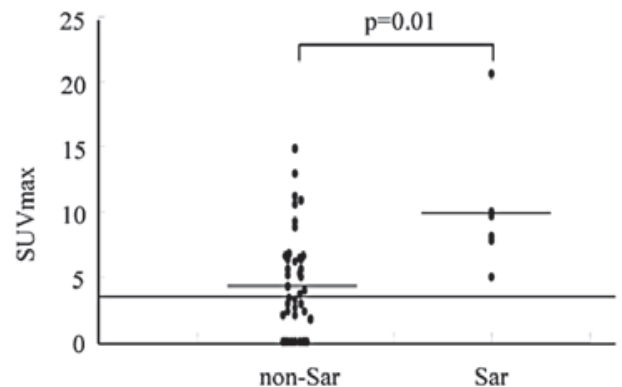

B

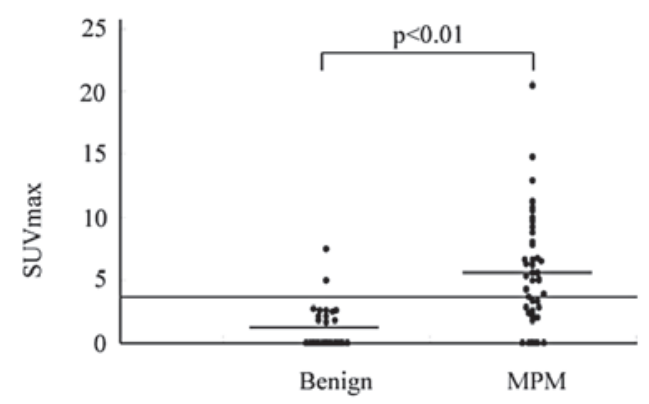

D

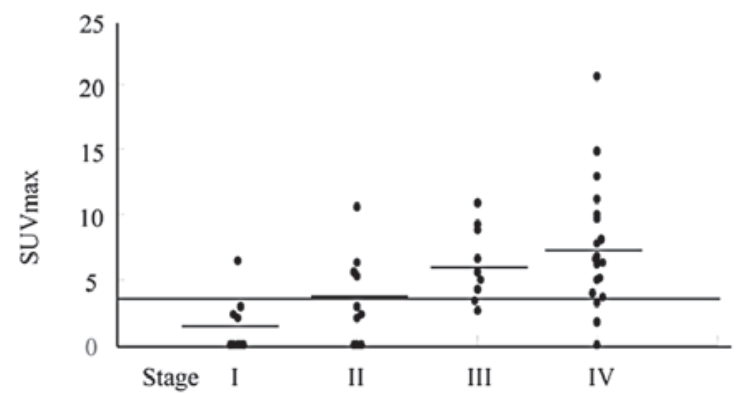

Figure 1. SUVmax levels in patients with MPM and non-MPM patients. (A) Sensitivity and specificity of SUVmax levels for distinguishing patients with MPM from non-MPM patients (ROC curve). An analysis that included 47 MPM patients and 29 non-MPM subjects revealed an AUC of 0.803 (95\% CI, 0.722-0.885). At a cut-off value of 3.5, the diagnostic sensitivity was 59.6\%, and the specificity was 93.1\%. (B) SUVmax levels in non-MPM patients and MPM patients were measured as described in Materials and methods. (C) SUVmax levels between the different MPM histological groups were measured as described in Materials and methods. Sar, sarcomatoid subtype. (D) SUVmax levels for MPM patients divided into four stages are shown. The nonparametric Mann-Whitney U-test (B and C) or the nonparametric Kruskal-Wallis test followed by the Mann-Whitney U-test (D) was used. $\mathrm{p}<0.05$ was considered significant. Horizontal bars represent the mean value of each group. The cut-off value is shown as a horizontal line.

sarcomatoid, $4.6 \pm 3.9$ and sarcomatoid, $10.2 \pm 5.4$, respectively) $(\mathrm{p}=0.01)$ (Fig. 1C). Moreover, scatter plots of SUVmax levels in MPM demonstrated tendencies to increase with increasing stage (stage I, 1.5 \pm 2.2 ; stage II, 3.5 \pm 3.4 ; stage III, $6.3 \pm 2.8$; and stage IV, 7.6 \pm 4.9$)(\mathrm{p}<0.01$ by the nonparametric KruskalWallis test followed by the Mann-Whitney U-test) (Fig. 1D). However, there were no significant differences in SUVmax levels by gender (male, 5.2 \pm 4.4 and female, 5.5 \pm 4.7 ) and age $(\leq 65,5.5 \pm 4.3$ and $>65$ years, $5.1 \pm 4.7)$. There were no significant differences in SUVmax levels between patients with benign asbestos pleurisy and those with non-MPM (1.8 \pm 1.5 and $1.1 \pm 1.8$, respectively).

Relationship between SUVmax levels and overall survival. We were able to closely monitor all MPM patients for 700 days. To study the relationship between SUVmax levels and patient clinical course, we divided patients based on their SUVmax levels at the time of the first measurement. The first group included patients with SUVmax levels $<3.5$, the cut-off value that was used. In this group of 19 patients, the mean SUVmax level was $1.4 \pm 1.4$. The other group included the remaining 28 patients with SUVmax levels $\geq 3.5$, whose mean SUVmax level was $8.0 \pm 3.7$. The difference in overall survival between the groups with SUVmax levels lower and higher than 3.5 was significant ( $\mathrm{p}=0.02)$ (Fig. 2).

Cox's regression analysis was carried out for all MPM patients for whom data on age, gender, histology, performance status, and SUVmax levels were available. An independent significant prognostic effect on survival of age $(\leq 65$ versus $>65$; HR, 2.4; 95\% CI, 1.07-5.23; $\mathrm{p}=0.03$ ) was found.

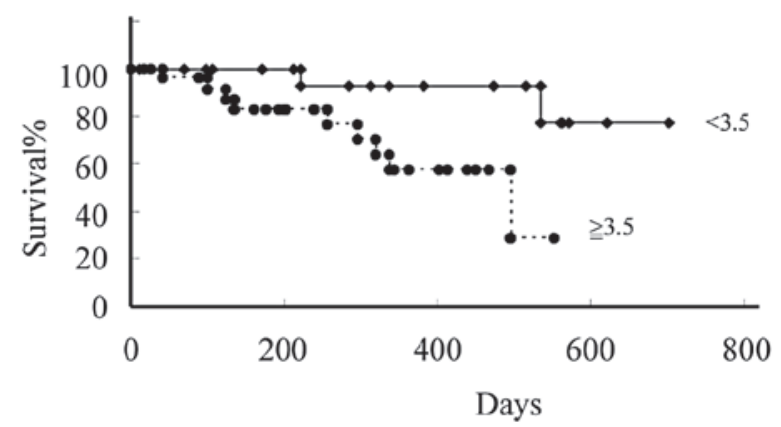

Figure 2. Survival of MPM subjects according to SUVmax levels $(<3.5$ or $\geq 3.5$ ). Estimates of the probability of survival were calculated using the Kaplan-Meier method and compared using the log-rank test.

\section{Discussion}

Mesothelioma is a malignant transformation caused by the exposure of mesothelial cells to asbestos, and has a limited response to conventional therapy, and a very poor prognosis. The lifetime risk of mesothelioma is associated with occupational and/or environmental asbestos exposure history. Due to the long latency period (typically longer than 30 years) between first asbestos exposure and the onset of the disease, the diagnosis of mesothelioma remains difficult with an increasing incidence worldwide (1-3).

Current imaging tools lack the ability to accurately detect the distribution of MPM. Previous reports have shown that $\mathrm{CT}$ and magnetic resonance imaging (MRI) provide anatomic 
information that is not precise in the preoperative staging and resectability of MPM $(17,18)$.

PET is a very useful imaging tool for the clinical assessment of cancer patients (9). The uptake of $18 \mathrm{~F}-\mathrm{FDG}$ is commonly higher in most types of cancer than that in most normal organs. Bénard et al examined whether PET helps to distinguish between benign and malignant pleural disease and revealed that PET was a sensitive tool in 22 MPM patients. The main results of their study were that differentiation of benign lesions from malignant pleural diseases still required pathological confirmation and that PET did seem to be useful in guiding surgical biopsy (12). In the next report, they performed a survival analysis of 17 MPM patients (13), however, its clinical utility in MPM was not fully investigated. In this study, we examined the diagnostic and prognostic role of PET in 47 Japanese MPM patients. Our current study was more than double the size of the prior study in a single institution. 18F-FDG uptake levels are semi-quantitatively expressed as SUVmax levels in general clinical institutions. Similarly to Bénard et al, we found that patients with MPM had significantly higher SUVmax levels than the non-MPM population containing patients with a history of asbestos exposure and a group that had never been exposed to asbestos. The difference in SUVmax levels between the population with or without a history of asbestos exposure was not significant, suggesting that not only asbestos exposure, but also MPM growth is required for elevations in SUVmax levels. Furthermore, we analyzed SUVmax levels of MPM patients using histology and staging systems and we found significant differences in SUVmax levels among MPM histological groups and staging. Although pleural biopsies such as VATS are often critical to the diagnosis of MPM, they are invasive procedures. Therefore, new non-invasive techniques for assessment of MPM are required to judge whether those procedures should be practiced for diagnosis of MPM.

Although the diagnostic sensitivity and NPV of SUVmax levels for MPM measured on a ROC curve were not high (59.6 and 58.7\%, respectively), its specificity and PPV was fairly high (93.1 and $93.3 \%$, respectively), suggesting that high SUVmax levels are supportive of a differential diagnosis of MPM, which is extremely difficult to obtain for individuals with pleural disease.

Moreover, we also demonstrated a significant correlation between SUVmax levels and survival in most MPM patients using the Kaplan-Meier method, which suggested their usefulness as a tool to estimate prognosis. Since there is no clinical useful imaging system of MPM and early distinction of MPM patients from those with benign asbestos-related diseases is necessary, we propose that measuring SUVmax levels is a useful imaging tool for the clinical management of MPM.

In conclusion, we demonstrated that patients with MPM had significantly higher SUVmax levels than a non-MPM population with or without a history of asbestos exposure, and the Kaplan-Meier method revealed a significant correlation between SUVmax levels of MPM patients and survival. It is suggested that SUVmax levels are a novel useful diagnostic and prognostic imaging tool for MPM.

\section{Acknowledgements}

We thank Ms. Hidemi Kitai for providing technical assistance. This work was supported by grants from KAKENHI, a Grantin-Aid for Scientific Research (C) (23591167) and Health Labour Sciences Research Grant.

\section{References}

1. Robinson BW, Musk AW and Lake RA: Malignant mesothelioma. Lancet 366: 397-408, 2005.

2. Robinson BW and Lake RA: Advances in malignant mesothelioma. N Engl J Med 353: 1591-1603, 2005.

3. Wagner JC, Sleggs CA and Marchand P: Diffuse pleural mesothelioma and asbestos exposure in the North Western Cape Province. Br J Ind Med 17: 260-271, 1960.

4. Selikoff IJ, Hammond EC and Seidman H: Latency of asbestos disease among insulation workers in the United States and Canada. Cancer 15: 2736-2740, 1980.

5. Vogelzang NJ, Rusthoven JJ, Symanowski J, et al: Phase III study of pemetrexed in combination with cisplatin versus cisplatin alone in patients with malignant pleural mesothelioma. J Clin Oncol 21: 2636-2644, 2003.

6. Nowak AK, Lake RA, Kindler HL, et al: New approaches for mesothelioma: biologics, vaccines, gene therapy, and other novel agents. Semin Oncol 29: 82-96, 2002.

7. Leung AN, Müller NL and Miller RR: CT in differential diagnosis of diffuse pleural disease. AJR Am J Roentgenol 154: 487-492, 1990.

8. Müller NL: Imaging of the pleura. Radiology. 186: 297-309, 1993.

9. Rigo P, Paulus P, Kaschten BJ, et al: Oncological applications of positron emission tomography with fluorine-18 fluorodeoxyglucose. Eur J Nucl Med 23: 1641-1674, 1996.

10. Gupta NC, Frank AR, Dewan NA, et al: Solitary pulmonary nodules: detection of malignancy with PET with 2-[F-18]-fluoro2-deoxy-D-glucose. Radiology 184: 441-444, 1992.

11. Patz EF Jr, Lowe VJ, Hoffman JM, et al: Focal pulmonary abnormalities: evaluation with F-18 fluorodeoxyglucose PET scanning. Radiology 188: 487-490, 1993.

12. Bénard F, Sterman D, Smith RJ, et al: Metabolic imaging of malignant pleural mesothelioma with fluorodeoxyglucose positron emission tomography. Chest 114: 713-722, 1998.

13. Bénard F, Sterman D, Smith RJ, et al: Prognostic value of FDG PET imaging in malignant pleural mesothelioma. J Nucl Med 40: 1241-1245, 1999.

14. Flores RM, Akhurst T, Gonen M, et al: Positron emission tomography predicts survival in malignant pleural mesothelioma. J Thorac Cardiovasc Surg 132: 763-768, 2006.

15. Yildirim H, Metintas M, Entok E, et al: Clinical value of fluorodeoxyglucose-positron emission tomography/computed tomography in differentiation of malignant mesothelioma from asbestos-related benign pleural disease: an observational pilot study. J Thorac Oncol 4: 1480-1484, 2009.

16. Rusch VW: A proposed new international TNM staging system for malignant pleural mesothelioma. From the International Mesothelioma Interest Group. Chest 108: 1122-1128, 1995.

17. Heelan RT, Rusch VW, Begg CB, et al: Staging of malignant pleural mesothelioma: comparison of CT and MR imaging. AJR Am J Roentgenol 172: 1039-1047, 1999.

18. Patz EF Jr, Shaffer K, Piwnica-Worms DR, et al: Malignant pleural mesothelioma: value of CT and MR imaging in predicting resectability. AJR Am J Roentgenol 159: 961-966, 1992. 